\title{
Noise-induced suppression of resonant vibrations
}

\author{
Roman V. Bobryk • Daniil Yurchenko
}

Received: 3 October 2013 / Revised: 16 October 2013 / Accepted: 17 October 2013 / Published online: 2 November 2013

(C) Springer-Verlag Berlin Heidelberg 2013

\begin{abstract}
The effect of random parametric noises on reduction of a resonant amplitude of forced linear SDOF systems is explored. It is found that noise-induced excitations of the stiffness coefficient of the system can substantially reduce the amplitude of resonant vibrations. Three different sources of random noises with an exponential correlation function are used to demonstrate the effect.
\end{abstract}

Keywords Vibration suppression - Parametric resonance . Bounded noise $\cdot$ Exponential correlation function . Closure method

\section{Introduction}

It has been noted that changing a stiffness of a linear system may lead to increase or decrease in the system's amplitude [1]. Later, a similar effect was described in the paper [2], whereas the possible destabilizing effect, leading to the system instability was reported in paper [3]. Since then the topic of a damping effect of a parametrically changing stiffness attracts scientists and a number of publications has significantly grown in the last decade. In [4] the authors discuss the possibility of using the parametric suppression of self-excited oscillations of a mechanical system excited by incoming flow. In series of papers [5-9] the authors discuss application of parametric suppression of oscillations of para-

\section{R. V. Bobryk}

Department of Mathematics and Natural Sciences,

Jan Kochanowski University, Kielce, Poland

D. Yurchenko $(\bowtie)$

Department of Mechanical Engineering,

Heriot-Watt University, Edinburgh, UK

e-mail: d.yurchenko@hw.ac.uk metrically excited systems. Recently in review paper [10] experimental results are presented, which verified the damping effect. It also has been confirmed that the most significant amplitude reduction is achieved for the lowest modes.

All of the above mentioned publications assumed deterministic changes in the system's stiffness. However, it has been shown in $[11,12]$ that a stochastic excitation may also lead to the suppression of the oscillation amplitude. The steady state response amplitude $A$ of a SDOF linear system

$\ddot{x}+2 \gamma \dot{x}+\omega^{2} x=a \sin (\Omega t), \quad \gamma>0$,

has the form

$A=\frac{a}{\sqrt{\left(\omega^{2}-\Omega^{2}\right)^{2}+4 \gamma^{2} \Omega^{2}}}$,

and it manifests the resonance phenomenon if the viscous damping constant $\gamma$ is small and $\omega \approx \Omega$ [13]. The phenomenon is very important and can be observed in a broad class of systems [14]. Usually, a suppression of the resonant oscillations may be achieved by increasing the viscous damping constant $\gamma$. In the case of a light damping constant another method for the resonant vibrations reduction was proposed in $[11,12]$. It relies on an introduction of a random excitation of the natural frequency $\omega$. More precisely, the system (1) is replaced by the following stochastic system:

$\ddot{y}+2 \gamma \dot{y}+\omega^{2}[1+\sigma \xi(t)] y=a \sin (\Omega t)$,

where $\xi(t)$ is a zero-mean stationary random noise.

Let $A_{\text {mean }}$ and $G_{m}=A_{\text {mean }} / A$ be the amplitude of the long-term behavior of the mean $E[y(t)]$ and the mean amplitude ratio, respectively. It was shown in $[11,12]$ that if the noise intensity $\sigma$ is sufficiently small, then the mean amplitude ratio is less than one provided the spectral density $S(z)$ of the noise is a continuous decreasing function on $z \geq 0$. However if the noise intensity is small, then the deviation of 
the mean amplitude ratio from one is small too and so is the reduction. Can a grater deviation be obtained? Here we try to answer this question.

If the noise intensity is not small then the solutions of Eq. (2) may be unstable. Note that in the undamped case the solutions are almost sure exponentially increasing if $t \rightarrow \infty$. If the process $\xi(t)$ in Eq. (2) is a Gaussian white noise then the mean $E[y(t)]$ satisfies the Eq. (1) and thereby $G_{m}$ is one. Therefore colored noise models of $\xi(t)$ should be considered. On the other hand, the ratio $G_{m}$ may not be sufficient to estimate a real reduction. At least an analysis of the variance of the solution to Eq. (2) should be done.

In this paper we suppose that the noise has the correlation function

$$
K(t)=E[\xi(t+u) \xi(u)]=\exp (-\alpha|t|) .
$$

Three types of the random noises $\xi(t)$ with correlation (3) are considered. The first one is the well known OrnsteinUhlenbeck (OU) process [15]. The second one is the sineWiener (SW) process:

$\xi(t)=\sqrt{2} \sin [\theta+\sqrt{2 \alpha} w(t)]$,

with $w(t)$ standing for the standard Wiener process, $\theta$ is an uniformly distributed in $[0,2 \pi]$ random variable independent of $w(t)$. This process is a special case of the so-called bounded noise $\beta \sin [\theta+\mu t+\sqrt{2 \alpha} w(t)]$ which has recently been widely used in the modeling of mechanical systems [16-19]. The third one is the well known telegraphic (TG) process [15] with two states $\{-1,1\}$ and the transition intensity $\alpha / 2$. This case is of special interest, since it resembles the case of optimal control of a system with a bounded in magnitude control force. Moreover, whereas in the control case the switching between two values happens at certain time instants, depending on the position/velocity of the system and the goal of the optimal control, in the switching systems it happens arbitrary [20]. Note that the SDOF systems with a random parametric noise has been the subject of many studies (see e.g. $[12,21,22]$ and references therein).

\section{Mean value calculations}

Let's consider Eq. (2) and denote the vector

$\mathbf{z}:=(\dot{y}, y)^{T}$,

which satisfies the following equation in $\mathbb{R}^{2}$ :

$\frac{d \mathbf{z}}{d t}=\mathbf{A z}+\xi(t) \mathbf{C z}+a \sin (\Omega t) \mathbf{b}$,

where $\mathbf{b}=(1,0)^{T}$,

$\mathbf{A}=\left(\begin{array}{cc}-2 \gamma & -\omega^{2} \\ 1 & 0\end{array}\right), \quad \mathbf{C}=\left(\begin{array}{cc}0 & -\omega^{2} \sigma \\ 0 & 0\end{array}\right)$
Let first consider the TG noise case. In this case one can obtain the following closed set of equations for the mean $E[\mathbf{z}(t)][23]:$

$\frac{d E[\mathbf{z}]}{d t}=\mathbf{A} E[\mathbf{z}]+\mathbf{C} \mathbf{z}_{1}+a \sin (\Omega t) \mathbf{b}$,

$\frac{d \mathbf{z}_{1}}{d t}=-\alpha \mathbf{z}_{1}+\mathbf{A} \mathbf{z}_{1}+\mathbf{C} E[\mathbf{z}]$,

where $\mathbf{z}_{1}=E[\xi(t) \mathbf{z}]$.

Unfortunately, a closed set of linear ordinary differential equations for the mean $E[\mathbf{z}(t)]$ cannot be obtained in $\mathrm{OU}$ and SW noise cases but one can get an infinite set of linear ordinary differential equations. To obtain this set in $\mathrm{OU}$ noise case we use the following Donsker-Furutsu-Novikov formula [24-26]:

$E[\xi(t) \mathbf{R}[\xi]]=\int K(t, s) E\left[\frac{\delta \mathbf{R}[\xi]}{\delta \xi(s)}\right] d s$,

where $\mathbf{R}[\xi]$ is a functional of zero-mean Gaussian process $\xi(t)$ with the correlation function $K(t, s)$ and $\frac{\delta}{\delta \xi(s)}$ is an operator of the functional derivative (Volterra-Fréchet functional derivative). Applying this formula to the Eq. (5) we obtain the following infinite chain of equations (see $[27,28]$ for details):

$$
\begin{aligned}
& \frac{d E[\mathbf{z}]}{d t}=\mathbf{A} E[\mathbf{z}]+\mathbf{C} \mathbf{z}_{1}+a \sin (\Omega t) \mathbf{b}, \\
& \frac{d \mathbf{z}_{1}}{d t}=-\alpha \mathbf{z}_{1}+\mathbf{A} \mathbf{z}_{1}+\mathbf{C} \mathbf{z}_{2}+\mathbf{C} E[\mathbf{z}] . \\
& \frac{d \mathbf{z}_{k}}{d t}=-\alpha k \mathbf{z}_{k}+\mathbf{A} \mathbf{z}_{k}+\mathbf{C} \mathbf{z}_{k+1}+k \mathbf{C} \mathbf{z}_{k-1}, \quad k=2,3, \ldots
\end{aligned}
$$

Here

$$
\begin{aligned}
& \mathbf{z}_{k}(t):=\int_{0}^{t} \ldots \int_{0}^{t} \exp \left\{-\alpha \sum_{j=1}^{k}\left(t-s_{j}\right)\right\} \\
& \times E\left[\frac{\delta^{k} \mathbf{z}(t)}{\delta \xi\left(s_{1}\right) \cdots \delta \xi\left(s_{k}\right)}\right] d s_{1} \cdots d s_{k}, \quad k=1,2,3, \ldots,
\end{aligned}
$$

where

$$
\frac{\delta^{k} \mathbf{z}(t)}{\delta \xi\left(s_{1}\right) \cdots \delta \xi\left(s_{k}\right)}
$$

is the functional derivative of $k$ th order.

Let's consider now the SW noise case. The solution of Eq. (5) is a functional of the Wiener process and therefore we write $\mathbf{z}(t)=\mathbf{z}(t ; w(s))$. Using the Cameron-Martin formula [29] for the density of the Wiener measure under translation one can deduce that for all nonrandom $\lambda$,

$$
\begin{aligned}
& E[\exp \{i \lambda w(t)\} \mathbf{z}(t ; w(s))] \\
& \quad=\exp \left\{-\frac{\lambda^{2} t}{2}\right\} E[\mathbf{z}(t ; w(s)+i \lambda s)], \quad i=\sqrt{-1} .
\end{aligned}
$$


Since

$$
\begin{aligned}
& \sin (\theta+\sqrt{2 \alpha} w(t)) \\
& =\frac{\exp \{i \theta+i \sqrt{2 \alpha} w(t)\}-\exp \{-i \theta-i \sqrt{2 \alpha} w(t)\}}{2 i},
\end{aligned}
$$

we obtain the following infinite chain for $E[\mathbf{z}(t)]$ (see $[30,31]$ for details):

$\frac{d E[\mathbf{z}]}{d t}=\mathbf{A} E[\mathbf{z}]+\mathbf{C} \mathbf{z}_{1}+a \sin (\Omega t) \mathbf{b}$,

$\frac{d \mathbf{z}_{1}}{d t}=-\alpha \mathbf{z}_{1}+\mathbf{A} \mathbf{z}_{1}+\mathbf{C} \mathbf{z}_{2}+\frac{1}{2} \mathbf{C} E[\mathbf{z}]$,

$\frac{d \mathbf{z}_{k}}{d t}=-\alpha k^{2} \mathbf{z}_{k}+\mathbf{A} \mathbf{z}_{k}+\mathbf{C} \mathbf{z}_{k+1}+\frac{1}{4} \mathbf{C} \mathbf{z}_{k-1}, \quad k=2,3, \ldots$

Here

$$
\begin{gathered}
\mathbf{z}_{k}(t):=\frac{1}{(\sqrt{2}: i)^{k}} \exp \left\{-k^{2} \alpha t\right\}(E[\mathbf{z}(t ; w(s)+i k \sqrt{2 \alpha} s)] \\
\left.+(-1)^{k} E[\mathbf{z}(t ; w(s)-i k \sqrt{2 \alpha} s)]\right), \quad k=1,2,3, \ldots,
\end{gathered}
$$

where $\mathbf{z}(t ; w(s) \pm i k \sqrt{2 \alpha} s)$ is the solution of Eq. (5) with $w(t)$ replaced by $w(t) \pm i k t \sqrt{2 \alpha} t, i=\sqrt{-1}$.

Unlike the TG noise case, the chains are not closed in both SW and OU noise cases and we have to consider their closure. A natural closure scheme relies on truncation of the term $\mathbf{z}_{n+1}$ in the $(n+1)$ th equation of the chains $(9,10)$. The scheme is quickly convergent in both OU and SW noise cases [31,32].

After this closure, one obtains the following closed system of linear ordinary differential equations:

$$
\begin{aligned}
\frac{d \mathbf{z}_{0 n}}{d t}= & \mathbf{A} \mathbf{z}_{0 n}+\mathbf{C} \mathbf{z}_{1 n}+a \sin (\Omega t) \mathbf{b}, \\
\frac{d \mathbf{z}_{k n}}{d t}= & -\alpha d_{k} \mathbf{z}_{k n}+\mathbf{A} \mathbf{z}_{k n}+p_{k} \mathbf{C} \mathbf{z}_{k+1, n} \\
& +q_{k} \mathbf{C} \mathbf{z}_{k-1, n}, \quad k=1, \ldots, n-1, \\
\frac{d \mathbf{z}_{n n}}{d t}= & -\alpha d_{n} \mathbf{z}_{n n}+\mathbf{A} \mathbf{z}_{n n}+q_{n} \mathbf{C} \mathbf{z}_{n-1, n},
\end{aligned}
$$

where in the case of OU noise $d_{k}=k, p_{k}=1, q_{k}=k, k=$ $1,2,3, \ldots, n$, but in the case of SW noise $d_{1}=p_{1}=1$, $q_{1}=\frac{1}{2}, d_{k}=k^{2}, p_{k}=1, q_{k}=\frac{1}{4}, k=2,3, \ldots, n$. Of course the closed system (6) for the TG noise case can be considered as a special case of the system (12) with $p_{1}=$ $0, d_{1}=q_{1}=1, d_{k}=p_{k}=q_{k}=0, k=2,3, \ldots, n$.

One can rewrite the system (12) as the following linear differential equation in $\mathbb{R}^{2 n+2}$ :

$\frac{d \mathbf{z}_{n}}{d t}=\mathbf{P}_{n} \mathbf{z}_{n}+a \sin (\Omega t) \mathbf{d}_{n}$,

where $\mathbf{P}_{n}$ is a coefficient matrix of the system (12) and $\mathbf{d}_{n}$ is a $2(n+1) \times 1$ vector, $\mathbf{d}_{n}=(1,0, \ldots, 0)^{T}$.

Under condition of asymptotic stability of Eq. (12) with $a=0$, its long-time solution is

$\mathbf{z}_{n}(t)=\mathbf{a}_{n} \sin (\Omega t)+\mathbf{b}_{n} \cos (\Omega t)$
Then after substitution (13) to Eq. (12) we obtain for $\mathbf{a}_{n}$ and $\mathbf{b}_{n}$ the following expressions:

$\mathbf{b}_{n}=-a \Omega\left(\Omega^{2} \mathbf{I}_{2(n+1)}+\mathbf{P}_{n}^{2}\right)^{-1} \mathbf{d}_{n}, \quad \mathbf{a}_{n}=\mathbf{P}_{n} \mathbf{b}_{n} / \Omega$,

where $\mathbf{I}_{j}$ is a $j \times j$ unit matrix.

Therefore, an approximation for the amplitude $A_{\text {mean }}$ of the long-time behavior of the mean is

$A_{\text {mean }} \approx \sqrt{a_{n}^{2}(2)+b_{n}^{2}(2)}$

where $a_{n}(2)$ and $b_{n}(2)$ are the second element of the vector $\mathbf{a}_{n}$ and $\mathbf{b}_{n}$, respectively. In the case of TG noise the expression (15) for $n=1$ gives an exact value of the amplitude. It can be represented in the simpler form [21]

$A_{\text {mean }}=\sqrt{\frac{f_{1}^{2}+f_{2}^{2}}{f_{3}^{2}+f_{4}^{2}}}$,

with

$$
\begin{aligned}
f_{1}= & 2(\alpha+\gamma) a \Omega, \quad f_{2}=\left(\Omega^{2}-\omega^{2}-\alpha^{2}-2 \alpha \gamma\right), \\
f_{3}= & \left(\Omega^{2}-\omega^{2}\right)\left(\Omega^{2}-\omega^{2}-\alpha^{2}\right)-a \\
& -2\left(3 \alpha \omega+2 \gamma^{2}\right) \omega^{2}+2 \alpha \gamma \omega^{2}, \\
f_{4}= & 2 \Omega(\alpha+2 \gamma)\left(\omega^{2}-\Omega^{2}+\alpha \gamma\right) .
\end{aligned}
$$

The mean amplitude ratio $G_{m}$ in TG noise case is plotted in Fig. 1 (solid line) for the values $a=1, \omega=\Omega=$ $1, \gamma=0.05, \sigma=0.65, \alpha \in[0.001,0.5]$ and $D-$ a standard deviation, defined by Eq. (26). The ratio is also plotted

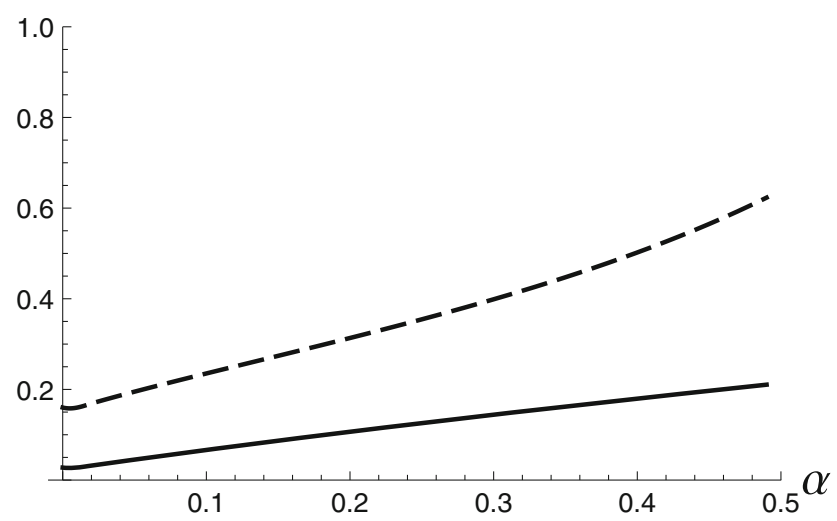

Fig. 1 The mean amplitude ratio $G_{m}$ (solid line) and the ratio $D / A$ (dashed line) in the TG noise case for the values $a=1, \omega=\Omega=$ $1, \gamma=0.05, \sigma=0.65, \alpha \in[0.01,0.5]$ 


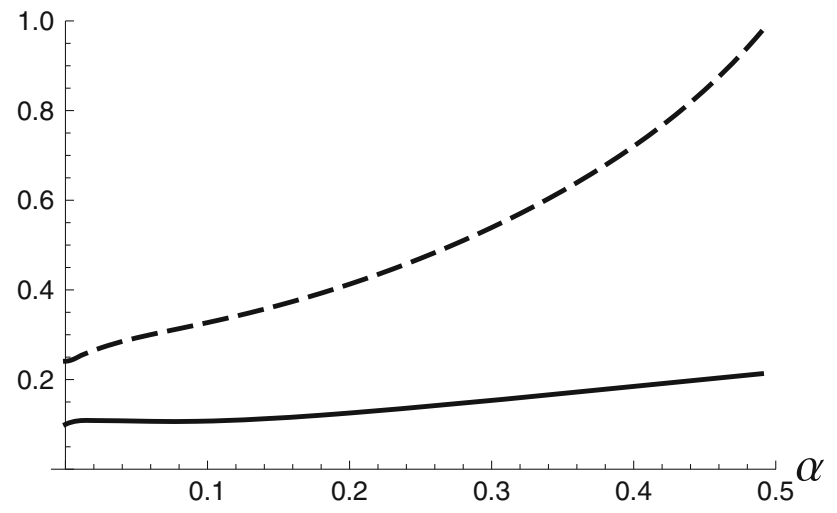

Fig. 2 The mean amplitude ratio $G_{m}$ (solid line) and the ratio $D / A$ (dashed line) in the SW noise case for the values $a=1, \omega=\Omega=$ $1, \gamma=0.05, \sigma=0.65, \alpha \in[0.01,0.5]$

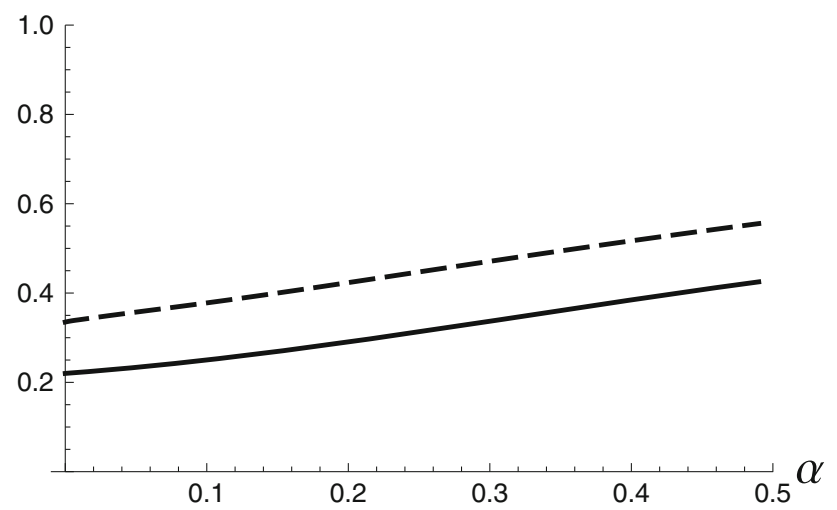

Fig. 3 The mean amplitude ratio $G_{m}$ (solid line) and the ratio $D / A$ (dashed line) in the OU noise case for the values $a=1, \omega=\Omega=1$, $\gamma=0.05, \sigma=0.4, \alpha \in[0.01,0.5]$

for the same values in the SW and OU cases in Figs. 2 and 3, respectively (solid line). Here the numerical computations of the expression (15) for the truncation index $n=40$ have been used. Note that for the truncation index $n=80$ we have the same plots.

\section{The second order moments}

However, the parameter $G_{m}$ may be useless if the solution of Eq. (2) has a large variance. To examine this issue consider the vector

$\mathbf{u}:=\left(\dot{y}^{2}, y \dot{y}, y^{2}\right)^{T}$.

The Eq. (2) implies that this vector satisfies the following equation in $\mathbb{R}^{3}$ :

$\frac{d \mathbf{u}}{d t}=\mathbf{A}_{\mathbf{1}} \mathbf{u}+\xi(t) \mathbf{C}_{\mathbf{1}} \mathbf{u}+a \sin (\Omega t) \mathbf{B}_{\mathbf{1}} \mathbf{z}$, where

$$
\begin{array}{ll}
\mathbf{A}_{1}=\left(\begin{array}{ccc}
-4 \gamma & -2 \omega^{2} & 0 \\
1 & -2 \gamma & -\omega^{2} \\
0 & 2 & 0
\end{array}\right), \\
\mathbf{C}_{\mathbf{1}}=\left(\begin{array}{ccc}
0 & -2 \omega^{2} \sigma & 0 \\
0 & 0 & -\omega^{2} \sigma \\
0 & 9 & 0
\end{array}\right), \quad \mathbf{B}_{1}=\left(\begin{array}{ll}
2 & 0 \\
0 & 1 \\
0 & 0
\end{array}\right) .
\end{array}
$$

In a similar way as it was done for the mean one obtains the following system for the vector of the second order moments:

$$
\begin{aligned}
\frac{d E[\mathbf{u}]}{d t}= & \mathbf{A}_{1} E[\mathbf{u}]+\mathbf{C}_{1} \mathbf{u}_{1}+a \sin (\Omega t) \mathbf{B}_{1} E[\mathbf{z}] \\
\frac{d \mathbf{u}_{1}}{d t}= & -\alpha \mathbf{u}_{1}+\mathbf{A}_{1} \mathbf{u}_{1}+p_{1} \mathbf{C}_{1} \mathbf{u}_{2}+q_{1} \mathbf{C}_{1} E[\mathbf{u}] \\
& +a \sin (\Omega t) \mathbf{B}_{1} \mathbf{z}_{1}, \\
\frac{d \mathbf{u}_{k}}{d t}= & -\alpha d_{k} \mathbf{u}_{k}+\mathbf{A}_{1} \mathbf{u}_{k}+p_{k} \mathbf{C}_{1} \mathbf{u}_{k+1}+q_{k} \mathbf{C}_{1} \mathbf{u}_{k-1} \\
& +a \sin (\Omega t) \mathbf{B}_{1} \mathbf{z}_{k}, \quad k=2,3, \ldots
\end{aligned}
$$

The chain (19) has only two equations in the TG noise case $\left(p_{1}=0, q_{1}=1\right)$.

In the OU and SW cases the chain is not closed and using the natural closure procedure in chain (19) one obtains the following closed system:

$$
\begin{aligned}
\frac{d \mathbf{u}_{0 n}}{d t}= & \mathbf{A}_{1} \mathbf{u}_{0 n}+\mathbf{C u}_{1 n}+a \sin (\Omega t) \mathbf{B}_{1} \mathbf{z}_{0 n} \\
\frac{d \mathbf{u}_{k n}}{d t}= & -\alpha d_{k} \mathbf{u}_{k n}+\mathbf{A}_{1} \mathbf{z}_{k n}+p_{k} \mathbf{C}_{1} \mathbf{z}_{k+1, n}+q_{k} \mathbf{C}_{1} \mathbf{u}_{k-1, n} \\
& +a \sin (\Omega t) \mathbf{B}_{1} \mathbf{z}_{k n}, \quad k=1,2, \ldots, n-1 \\
\frac{d \mathbf{u}_{n n}}{d t}= & -\alpha d_{n} \mathbf{u}_{n n}+\mathbf{A}_{1} \mathbf{u}_{n n}+q_{n} \mathbf{C z}_{n-1, n} \\
& +a \sin (\Omega t) \mathbf{B}_{1} \mathbf{z}_{n n} .
\end{aligned}
$$

One can represent the system (20) as the following system in $\mathbb{R}^{3 n+3}$ :

$\frac{d \mathbf{u}_{n}}{d t}=\mathbf{Q}_{n} \mathbf{u}_{n}+a \sin (\Omega t) \mathbf{D}_{n} \mathbf{z}_{n}$,

where $\mathbf{Q}_{n}$ and $\mathbf{D}_{n}$ are the matrices coefficients of system (20). Under condition of asymptotic stability of Eq. (21) with $a=0$, its long-time solution is

$\mathbf{u}_{n}(t)=\mathbf{g}_{n}+\mathbf{r}_{n} \sin (\Omega t)+\mathbf{s}_{n} \cos (\Omega t)$.

After substitution (13) and (22) to Eq. (21) one obtains the following expressions for the coefficients of (22):

$\mathbf{g}_{n}=-a \mathbf{Q}_{n}^{-1} \mathbf{a}_{n} \mathbf{b}_{n}$,

$\mathbf{s}_{n}=a\left(4 \Omega_{3 n+3}^{\mathbf{I}}+\mathbf{Q}_{n}^{2}\right)^{-1}\left(\mathbf{Q}_{n} \mathbf{D}_{n} \mathbf{a}_{n}-2 \Omega \mathbf{D}_{n} \mathbf{b}_{n}\right) / 2$,

$\mathbf{r}_{n}=\left(2 \mathbf{Q}_{n} \mathbf{s}_{n}-a \mathbf{D}_{n} \mathbf{a}_{n}\right) /(4 \Omega)$.

It follows from (13) to (14) and (22) to (23) that the longtime variance of the solution to Eq. (2) can be approximated by the following expression: 


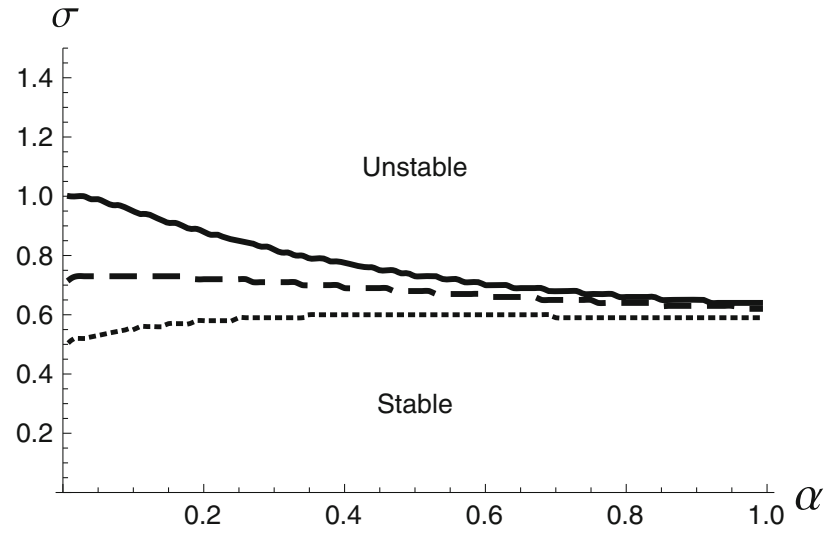

Fig. 4 Energetic stability diagram to the Eq. (2) for the values $a=$ $1, \gamma=0.05, \omega=\Omega=1, \alpha \in[0.001,1]$ and $\sigma \in[0,1.5]$. Solid, dashed and dotted curves separate stability and instability regions for TG, SW and OU noise cases, respectively

$V_{n}=g_{n}(3)-\frac{a_{n}(2)^{2}}{2}-\frac{b_{n}(2)^{2}}{2}+c_{n} \sin (2 \Omega t+\phi)$,

where $\phi$ is some phase,

$c_{n}=\sqrt{\left(r_{n}(3)-a_{n}(2) b_{n}(2)\right)^{2}+\left(s_{n}(3)+\frac{a_{n}(2)^{2}}{2}-\frac{b_{n}(2)^{2}}{2}\right)^{2}}$,

$g_{n}(3), r_{n}(3)$ and $s_{n}(3)$ are the third elements of the vectors $\mathbf{g}_{n}, \mathbf{r}_{n}$ and $\mathbf{s}_{n}$, respectively. Therefore, the maximum value $D$ of the long-time standard deviation of the solution to Eq. (2) can be approximated by the following expression:

$D \approx \sqrt{g_{n}(3)-\frac{a_{n}(2)^{2}}{2}-\frac{b_{n}(2)^{2}}{2}+c_{n}}$.

For $n=1$ this formula is exact in the TG noise case and the ratio $D / A$ is plotted in Fig. 1 (dashed line). In the case of SW and OU noise cases the ratio $D / A$ is plotted in Figs. 2 and 3 for $n=40$ (dashed line).

The expression (25) is obtained under condition of asymptotic stability of Eq. (21) with $a=0$. In the TG noise case this condition is nothing but the mean square stability to Eq. (2). In the SW and OU cases we have good approximations if truncation index $n$ is sufficiently large. In Fig. 4 the mean square stability diagram is plotted in the TG (solid line), SW (dashed line) and OU (dotted line) noise cases. It has obtained by the numerical analysis of matrix eigenvalues [28,30].

It follows from Figs. 1, 2 and 3 that with probability close to one there are real suppressions of the resonant oscillations in the TG and SW noise cases if $\alpha \in[0,0.2]$ and $\alpha \in[0,0.1]$, respectively. However, in the OU noise case real suppressions may not be observed. This fact can be explained by unboundedness of Gaussian processes. Indeed, OU noise as a Gaussian process can take arbitrary large values with a positive probability. Therefore there is a possibil- ity of non-oscillatory behaviour of the solution to Eq. (2) if $1+\sigma \xi(t)<0$.

It follows also from Figs. 1, 2 and 3 that the minimum of the mean amplitude ratio $G_{m}$ and the ratio $D / A$ is achieved as $\alpha \rightarrow 0$. In this limit the TG random process tends to a random variable $\zeta$ which takes only two values 1 and -1 with the same probability. Then one can be easily convinced that the long-time solution of Eq. (2) in resonance case $(\omega=\Omega)$ is

$y(t)=\frac{a(\sigma \omega \zeta \sin (\omega t)-2 \gamma \cos (\omega t))}{4 \gamma^{2} \omega+\sigma^{2} \omega^{3} \zeta^{2}}$,

under the condition $\sigma \zeta>-1$. This condition is always fulfilled in TG noise case if $\sigma<1$. It follows from (23) that the real amplitude $A_{r}$ of the oscillation is non-random and

$A_{r}=\frac{a}{\omega \sqrt{4 \gamma^{2}+\sigma^{2} \omega^{2}}}$

Thus, the real amplitude ratio $G_{r}=A_{r} / A$ is

$G_{r}=\frac{2 \gamma}{\sqrt{4 \gamma^{2}+\omega^{2} \sigma^{2}}}$

and so, one can get substantial reductions of the resonant amplitude.

Since expression (26)

$A_{\text {mean }}=\frac{2 a \gamma}{4 \gamma^{2} \omega+\sigma^{2} \omega^{3}}$.

It is worth to note that

$G_{r}=G_{m} \sqrt{1+\frac{\omega^{2} \sigma^{2}}{4 \gamma^{2}}}>G_{m}$

and therefore the mean amplitude ratio $G_{m}$ may not be a sufficient estimation of the real amplitude ratio. The analysis has to be completed by the analysis of variance at least.

\section{Conclusion}

This paper considered the possibility of reducing the resonant vibrations amplitude of the forced light-damped SDOF system by the noise-induced excitation of the its natural frequency. The fluctuations are modeled by the TG noise, the SW noise and the OU noise with a zero mean and exponential correlation. It is shown that substantial suppressions can be obtained in the narrow-band case. The TG noise model gives the best results as compared to the other two cases. One can suppose that the more efficient reduction can be obtained if the correlation function is more slowly decreasing. 


\section{References}

1. Frolov KV (1965) Amplitude reduction of resonant systems by change of parameters. Mashinovedenie 3:38-42 (in Russian)

2. Leitmann G (1994) Semiactive control for vibration attenuation. J Intell Mater Syst Mater 5:841-846

3. Corless M, Leitmann G (1997) Destabilization via active stiffness. Dyn Control 7:263-268

4. Fatimah S, Verhulst F (2003) Suppressing flow-induced vibrations by parametric excitation. Nonlinear Dyn 23:275-297

5. Tondl A (2008) To the problem of self-excited vibration suppression. Eng Mech 15:297-308

6. Tondl A, Ecker H (2003) On the problem of self-excited vibration quenching by means of parametric excitation. Arch Appl Mech 72:923-932

7. Tondl A, Pust L (2011) Further application of parametric antiresonance. Eng Mech 18:157-165

8. Dohnal F (2007) Suppressing self-excited vibrations by synchronous and time-periodic stiffness and damping variation. J Sound Vib 306:136-152

9. Dohnal F, Verhulst F (2008) Averaging in vibration suppression by parametric stiffness excitation. Nonlinear Dyn 54:231-248

10. Dohnal F (2012) Experimental studies on damping by parametric excitation using electromagnets. Proc IMechE 226(8):2015-2027

11. Dimentberg MF, Frolov KV (1966) Vibrations of SDOF system under periodic external excitation and random changes of the natural frequency. Mashinovedenie 4:3-10 (in Russian)

12. Dimentberg MF (1988) Statistical dynamics of nonlinear and timevarying systems. Research Studies Press, Taunton

13. Rao SS (2011) Mechanical vibrations, 5th edn. Prentice Hall, New York

14. Nayfeh AH, Mook D (1979) Nonlinear oscillations. Wiley, New York

15. Horsthemke W, Lefever R (1984) Noise-induced transitions. Springer, New York

16. Rong HW, Wang X, Wei X, Fang T (2008) Erosion of safe basins in a nonlinear oscillator under bounded noise excitation. J Sound Vib 313:46-56
17. Feng ZH, Lan XJ, Zhu XD (2009) Explanation on the importance of narrow-band random excitation characters in the response of a cantilever beam. J Sound Vib 325:923-937

18. Gan C, Lei H (2011) Stochastic dynamical analysis of a kind of vibro-impact system under multiple harmonic and random excitations. J Sound Vib 330:2174-2184

19. Yurchenko D, Naess A, Alevras P (2013) Pendulum's rotational motion governed by a stochastic Mathieu equation. Probab Eng Mech 31:12-18

20. Liberzon D (2003) Switching in systems and control. Birkhaüser, Boston

21. Gitterman M (2005) The noisy oscillator. World Scientific, Singapore City

22. Ibrahim RA (2008) Parametric random vibration. Dover, New York

23. Morrison JA, McKenna J (1973) Analysis of some stochastic ordinary differential equations. SIAM-AMS Proc 6:97-162

24. Donsker M (1964) On function space integrals. In: Proceedings of conference on theory and applications of analysis in function space, Dedham, 1963. The M.I.T. Press, Cambridge, pp 17-30

25. Furutsu K (1963) On the statistical theory of electromagnetic waves in a fluctuating medium. J Res Natl Bur Stand D 67:303-323

26. Novikov EA (1965) Functionals and the random-force method in turbulence theory. Sov Phys JETP 20:1290-1294

27. Bobryk RV, Stettner L (1999) Mean square stabilization of linear systems by mean zero noise. Stoch Stoch Rep 67:169-179

28. Bobryk RV, Chrzeszczyk A, Stettner L (2005) A closure procedure for random vibration parametric resonance. J Vib Control 11:215223

29. Cameron RH, Martin WT (1944) Transformations of Wiener integrals under translations. Ann Math 45:386-396

30. Bobryk RV, Stettner L (2005) Moment stability for linear systems with a random parametric excitation. Syst Control Lett 54:781-786

31. Bobryk RV (2007) Closure method and asymptotic expansions for linear stochastic systems. J Math Anal Appl 329:703-711

32. Bobryk RV (1985) Cumulant-free closure of the moment equations for the solution of a system of linear differential equations with randomly perturbed coefficients. Ukrainian Math J 37:443-450 